\title{
Afectación de la Resistencia a la Flexión en Concretos Modificados con Reciclado de Concreto
}

\section{Involvement of the bending strength in concrete modified with recycled concrete}

\author{
Sandra Elodia Ospina Lozano \\ Unisalle, Colombia \\ seospina@unisalle.edu.co
}

Lucio Guillermo López Yépez

Unisalle, Colombia

luglopez@unisalle.edu.co

\author{
Jeinner Arley Carrillo Carrillo \\ Unisalle, Colombia \\ arley009@hotmail.com
}

Leidy Johanna Díaz Garzón

Unisalle, Colombia

johadiaz@hotmail.es

Fecha de recepción: 20 de enero de 2016 / Fecha de aprobación: 7 de abril de 2016

\section{RESUMEN}

La necesidad actual de buscar alternativas para disminuir el impacto ambiental por la explotación cada vez mayor de las canteras y aprovechar los materiales resultantes de los procesos de demolición, en este documento se presentan los resultados obtenidos en diferentes tipos de concretos tradicionales sustituyendo en ellos, agregados resultantes del proceso de reciclaje de concreto.

Para desarrollar este trabajo, se variaron las relaciones agua/cemento y se adicionaron fibras de acero en diferentes porcentajes, lo que condujo a obtener efectos directos sobre las propiedades físico-mecánicas de los concretos estudiados; como resultado de la investigación, se encontró que se puede realizar un diseño de mezcla tradicional de concreto con un factor de disminución en resistencia que oscila entre el 50\% al 75\% de la resistencia esperada, para esto se utilizó la norma de ensayo ASTM C39ASTM C78,NTC 673 (Icontec, 2010) e INV E -414-07 (Invías, 2007).

PALABRAS CLAVE: Reciclado de concreto, concretos modificado, Resistencia a la compresión, Resistencia a la flexión.

\section{ABSTRACT}

The current need for alternatives to reduce the environmental impact by increasing quarrying and use the resulting materials demolition processes, this paper the results are presented in different replacing traditional types of concrete on them, added resulting from the process of recycling concrete.

To develop this work the water / cement ratios were varied and steel fibers are added in different percentages, leading to obtain direct effects on physical and mechanical properties of concrete studied; as a result of the investigation it was found that a design can be made of traditional concrete mixture a decrease in resistance factor ranging from $50 \%$ to $75 \%$ of the expected resistance, for this test standard ASTM was used C39- ASTM C78 (NTC 6731 and INV e-414 to 073).

KEYWORDS: Recycling of concrete, modified concrete, compressive strength, bending strength. 


\section{INTRODUCCIÓN}

El uso de productos reciclados en la producción de nuevos materiales de construcción hoy es una necesidad al reconocer que los recursos naturales no renovables se están extingüiendo, pero esto deberá ser una razón motivadora para seguir creando obras de infraestructura de alta calidad, que con alternativas diferentes a las tradicionales se puedan lograr. Para esto, las nuevas propuestas que surgen a nivel mundial, inicialmente se enfrentan a superar el como deberán ser evaluadas. La normatividad existente, solo define las condiciones y especificaciones de como evaluar un material en particular, pero en muchas ocasiones, quizás estas formas, no pueden ser utilizadas de la misma manera para analizar los materiales alternativos.

En el caso de estudio, se evaluaron las propiedades de resistencia del concreto en su estado endurecido (resistencia a la compresión, flexión y tracción indirecta), tomando en cuenta las normativas nacionales NTC 673 e INV E -414-07 (ASTM C39- ASTM C78), y se observó el efecto en la resistencia a la flexión con las normas estudiadas, para esto se hizo uso de concreto producto de residuo de demolición de concreto como agregado grueso, teniendo en cuenta el efecto que produce este tipo de agregado, además se introdujo en la mezcla, fibras de acero en distintos porcentajes con el fin de modificar las propiedades del concreto en su resistencia a la flexión.

Los resultados finales, proporcionaron datos para fabricar pavimentos rígidos, los cuales pueden ser usados en ciclorutas, o vías de bajos niveles de tránsito, un nuevo material que puede ser gran utilidad para el Instituto Nacional de Vías en Colombia.

\section{OBJETIVOS}

\section{Objetivo General}

Evaluar el desempeño de dos mezclas de concreto con agregados reciclados, reforzadas con tres diferentes cuantías de un tipo de fibras de acero, con base en los parámetros de resistencia especificados por la normatividad del INVIAS ${ }^{1}$.

\section{Objetivos Específicos}

- Valorar la influencia de la inclusión de tres diferentes cuantías volumétricas de un tipo de fibras cortas de acero, en las resistencias a flexión y tracción indirecta del concreto, con agregados reciclados para dos relaciones agua cemento.
- Comparar las resistencias y los modos de falla de elementos prismáticos de concreto con agregados reciclados, reforzado con un tipo de fibras cortas de acero, obtenidas de ensayos a flexión de tres puntos con los ensayos a flexión de cuatro puntos.

- Determinar la relación entre la resistencia a flexión y la resistencia a tracción indirecta del concreto reciclado, reforzado con un tipo de fibras cortas de acero.

\section{METODOLOGÍA}

En esta investigación se estudiaron dos relaciones agua cemento (0.5 y 0.6$)$ y cuatro porcentajes de fibras $(0 \%, 0.5 \%, 1.0 \%$ y $1.5 \%)$ : con estas dos variables, se establecieron los tipos de mezclas mostrados en la Tabla 1, en donde se indica la relación agua cemento (a/c) que se va a evaluar, y cuál va a ser la cantidad de fibras de acero a utilizar.

Tabla 1. Dosificaciones utilizadas para evaluar 10 mezclas

\begin{tabular}{|c|c|c|c|}
\hline Mezcla No. & Relación A/C & Contenido de fibras (\%) & Sigla utilizada \\
\hline 1 & 0.5 & 0 & $0.5 \mathrm{~A} / \mathrm{C}-0 \% \mathrm{FA}$ \\
\hline 2 & 0.5 & 0.5 & $0.5 \mathrm{~A} / \mathrm{C}-0.5 \% \mathrm{FA}$ \\
\hline 3 & 0.5 & 1.0 & $0.5 \mathrm{~A} / \mathrm{C}-1.0 \% \mathrm{FA}$ \\
\hline 4 & 0.5 & 1,5 & $0.5 \mathrm{~A} / \mathrm{C}-1.5 \% \mathrm{FA}$ \\
\hline 5 & 0.6 & 0 & $0.6 \mathrm{~A} / \mathrm{C}-0 \% \mathrm{FA}$ \\
\hline 6 & 0.6 & 0.5 & $0.6 \mathrm{~A} / \mathrm{C}-0.5 \% \mathrm{FA}$ \\
\hline 7 & 0.6 & 1.0 & $0.6 \mathrm{~A} / \mathrm{C}-1.0 \% \mathrm{FA}$ \\
\hline 8 & 0.6 & 1,5 & $0.6 \mathrm{~A} / \mathrm{C}-1.5 \% \mathrm{FA}$ \\
\hline
\end{tabular}

*El contenido de fibras fue adicionado en peso a la mezcla de cada dosificación

De acuerdo con la tabla anterior las siglas utilizadas tienen los siguientes significados:

A/C: Relación agua cemento

FA: fibras de acero

Para evaluar la propiedad de estudio, se propone un número de muestras para cada una de las dosificaciones. Se fabricaron mezclas para ensayos de compresión (sin adición de fibras de acero), tracción indirecta, flexión con cargas a los tercios de la luz y flexión con carga en el centro de la luz, el desglose de cada probeta fabricada se presenta en la siguiente Tabla.

'INVIAS: Instituto nacional de vías 
Tabla 2. Cantidad de probetas fabricadas por cada ensayo y cada dosificación

\begin{tabular}{|c|c|c|c|c|c|c|}
\hline Mezcla No. & Relación A/C & $\begin{array}{l}\text { Contenido de } \\
\text { fibras (\%) }\end{array}$ & Compresión & $\begin{array}{l}\text { Flexión con carga en el } \\
\text { centro de la luz }\end{array}$ & $\begin{array}{l}\text { Flexión con cargas a los } \\
\text { tercios de la luz }\end{array}$ & Tracción indirecta \\
\hline 1 & \multirow{4}{*}{0.5} & 0 & 3 Cilindros & 3 Viguetas & 3 Viguetas & 3 Cilindros \\
\hline 2 & & 0.5 & - & 3 Viguetas & 3 Viguetas & 3 Cilindros \\
\hline 3 & & 1.0 & - & 3 Viguetas & 3 Viguetas & 3 Cilindros \\
\hline 4 & & 1,5 & - & 3 Viguetas & 3 Viguetas & 3 Cilindros \\
\hline 5 & \multirow{4}{*}{0.6} & 0 & 3 Cilindros & 3 Viguetas & 3 Viguetas & 3 Cilindros \\
\hline 6 & & 0.5 & - & 3 Viguetas & 3 Viguetas & 3 Cilindros \\
\hline 7 & & 1.0 & - & 3 Viguetas & 3 Viguetas & 3 Cilindros \\
\hline 8 & & 1,5 & - & 3 Viguetas & 3 Viguetas & 3 Cilindros \\
\hline
\end{tabular}

Posterior al planteamiento de las dosificaciones que se quieren estudiar, se define el diseño de la mezcla de concreto, y de acuerdo con la metodología propuesta por el comité ACI 211 (Committe ACI 211, 2002), se obtienen las cantidades de cada uno de los componentes de las mezclas a estudiar, ver tabla 3. En el momento del mezclado, se hizo corrección por humedad por el contenido de agua del agregado, y se determinaron todas las cantidades de la mezcla correspondiente a estas modificaciones.

\section{Tabla 3. Parámetros del diseño de mezcla}

\begin{tabular}{|c|c|c|}
\hline Componentes del concreto & $\mathrm{A} / \mathrm{C}=0.5$ & $\mathrm{~A} / \mathrm{C}=0.6$ \\
\hline Cemento $\left(\mathrm{Kg} / \mathrm{m}^{3}\right)$ & 376 & 313 \\
\hline Agua $($ Litros) & 188 & 188 \\
\hline Agregado reciclado $\left(\mathrm{Kg} / \mathrm{m}^{3}\right)$ & 762 & 762 \\
\hline Agregado fino $\left(\mathrm{Kg} / \mathrm{m}^{3}\right)$ & 616 & 661 \\
\hline
\end{tabular}

Posteriormente, se fabricaron las probetas de ensayo y fueron falladas a los 28 días de edad de curado, este curado fue hecho por inmersión, de acuerdo con el procedimiento establecido en la norma de ensayo INV-E-402-07 (Invías, 2007).

\section{DESARROLLO}

Para la fabricación de las probetas se utilizaron los siguientes materiales.

\section{Cemento}

El cemento utilizado fue un cemento Portland Tipo $1^{2}$ de acuerdo con la Norma Técnica Colombiana NTC-121 marca Cemex de uso general en el país, al cual no se le exigen propiedades especiales, con una densidad de $2.84 \mathrm{gr} / \mathrm{cm}^{3}$.

\section{Agua}

Para la fabricación del concreto, se utilizó agua potable del acueducto de la ciudad de Bogotá, Colombia, no se realizó ningún tipo de ensayo al agua utilizada, suponiendo que cumplía las características de composición orgánica y nivel de ph, para este estudio de caso se despreció la influencia del agua en la evaluación de las diferentes resistencias.

\section{Agregado}

Para este caso, la granulometría usada fue controlada, tomando como base la ideal de Bolomey, la cual es una de las más usadas en las dosificaciones del concreto (López-Gayarre, Serna, DomingoCabo, Serrano-López, \& López-Colina, 1994), dicha curva se presenta en la Figura 1.

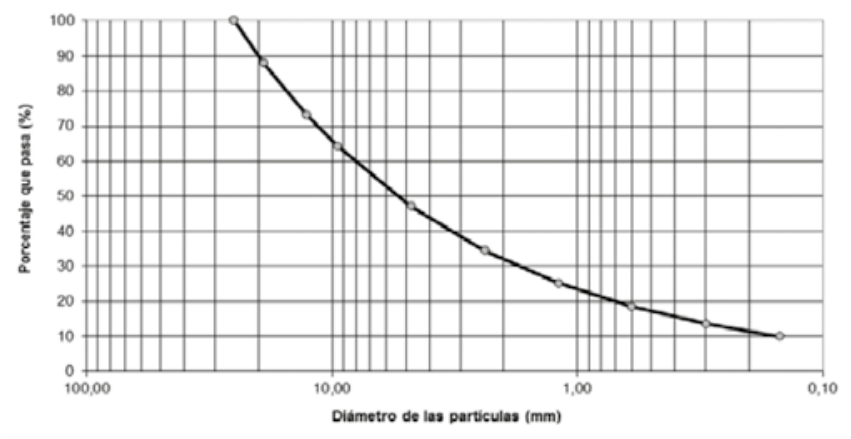

Figura 1. Curva ideal según Bolomey implementada en el material fabricado. (Carrillo \& Díaz, 2014)

En esta investigación, se utilizó agregado fino natural y se reemplazó en el 100\%, el agregado grueso natural por árido grueso, producto de residuo de demolición de concreto, en la Tabla 4 se muestra su caracterización.

${ }^{2}$ Clasificación dada en la NTC-121-82, donde se definen 5 tipos de cementos, el Tipo 1 presenta una resistencia a la compresión de cubos de mortero a los 28 días de $24 \mathrm{kgf} / \mathrm{cm}^{2}$. 
Tabla 4. Caracterización agregados utilizados para fabricar el concreto nuevo

\begin{tabular}{|c|c|c|}
\hline Propiedad & Fino Natural & Grueso reciclado \\
\hline Densidad nominal $\left(\mathrm{gr} / \mathrm{cm}^{3}\right)$ & 2.51 & 2.57 \\
\hline Densidad aparente $\left(\mathrm{gr} / \mathrm{cm}^{3}\right)$ & 1,99 & 2.18 \\
\hline Densidad aparente sss $\left(\mathrm{gr} / \mathrm{cm}^{3}\right)$ & 2.2 & 2.34 \\
\hline Absorción & 10.29 & 6.93 \\
\hline Masa unitaria suelta $\left(\mathrm{gr} / \mathrm{cm}^{3}\right)$ & 1.33 & 1,18 \\
\hline Masa unitaria apisonada $\left(\mathrm{gr} / \mathrm{cm}^{3}\right)$ & 1.47 & 1,27 \\
\hline Masa unitaria vibrada $\left(\mathrm{gr} / \mathrm{cm}^{3}\right)$ & 1.54 & 1,34 \\
\hline Humedad \% & 12.76 & 3,27 \\
\hline Módulo de finura $\%$ & 3.01 & - \\
\hline Tamaño máximo nominal & - & 1 in \\
\hline
\end{tabular}

\section{Fibras de acero}

Para esta investigación, se utilizaron fibras de acero con gancho (Figura 2), que cumplen con la especificación de fibras de acero para concreto reforzado con fibras norma ASTM-A820, cuyas características son resumidas en la Tabla 5.
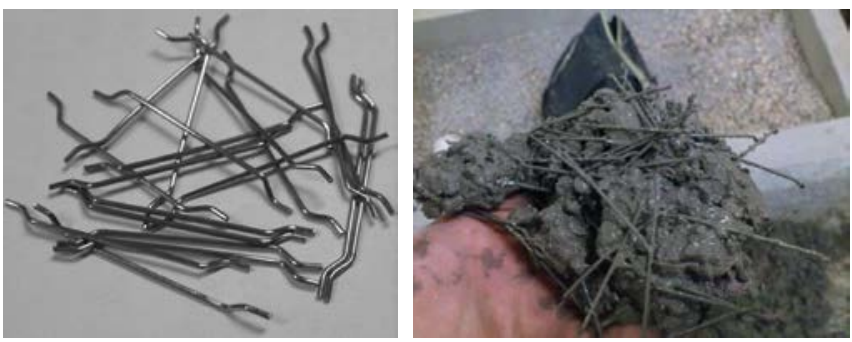

Figura 2. Fibras de acero utilizadas, (Carrillo \& Díaz, 2014)

Tabla 5. Caracterización de la fibra de acero utilizada para fabricar el concreto nuevo.

\begin{tabular}{|c|c|c|}
\hline Propiedad & Unidad & Valor \\
\hline Densidad real & $\mathrm{gr} / \mathrm{cm}^{3}$ & 7.86 \\
\hline Límite de resistencia a la tracción & $\mathrm{MPa}$ & 1100 \\
\hline Dimensiones & $\begin{array}{c}\varnothing 0.7 \mathrm{~mm} \times \\
\text { Long. } 25 \mathrm{~mm}\end{array}$ & \\
\hline
\end{tabular}

\section{RESULTADOS}

Los resultados obtenidos para los ensayos realizados son los siguientes.

\section{Ensayo a compresión}

Para evaluar la resistencia a la compresión, se siguió el procedimiento establecido en la norma de ensayo NTC 673 (ASTM C39). En la comparación de las resistencias para las dos relaciones A/C utilizadas, ocurrió una disminución de la resistencia del concreto reciclado, con respecto al valor teórico, de hasta el 36\% para la relación A/C de 0.6. Ver Tabla 6.

La disminución en la resistencia es atribuida a la sustitución del agregado de origen natural por el concreto reciclado, ya que los resultados dependen de la resistencia del concreto original que haya sido reciclado (Rammamurthy \& Gumaste, 1998) (Neha, 2013) de igual forma, esto también se ajusta a lo expresado por el comité ACI 555 el cual expone que la resistencia a compresión de concretos elaborados con agregados reciclados, tiene reducciones de un 15 - 40\% comparada con los concretos de agregado natural (Committe ACI 555, 2001). Coincidiendo esto con los resultados obtenidos en este estudio.

\section{Ensayo a Flexión con carga en el centro de la luz}

Para evaluar la resistencia a la flexión con carga en el centro de la luz se siguió el procedimiento establecido en la norma de ensayo INV E-415-07 (Invías, 2007), tal y como se muestra en la Figura 3.

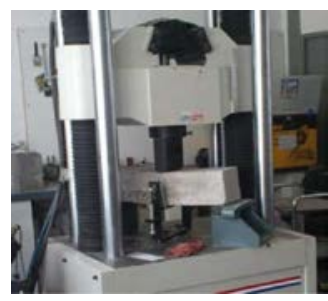

a)

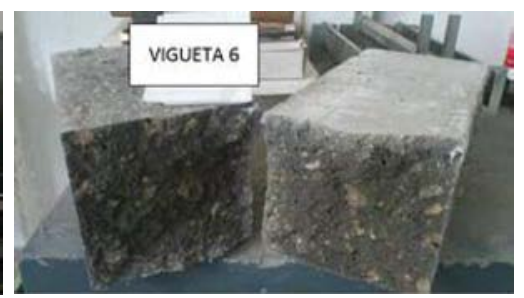

b)
Figura 3. Ensayo a flexión con carga en el centro de la luz. a) Montaje de las viguetas, b)Caras de una superficie de falla (agregado reciclado)

Tabla 6. Resultados para compresión inconfinada

\begin{tabular}{|c|c|c|c|c|}
\hline \multirow{2}{*}{ Relación A/C } & \multicolumn{2}{|c|}{ Resistencia a la compresión (MPa) } & \multirow{2}{*}{$\begin{array}{l}\% \text { de disminución de la } \\
\text { resistencia }\end{array}$} & \multirow{2}{*}{$\begin{array}{l}\text { Deformación máxima }(\mathrm{mm} / \mathrm{mm} \times \\
\qquad 10-4)\end{array}$} \\
\hline & Obtenida & Teórica (límite inferior)* & & \\
\hline 0.5 & 16.82 & 22.3 & 25 & 20 \\
\hline 0.6 & 10.11 & 15.79 & 36 & 11 \\
\hline
\end{tabular}

* Datos obtenidos de la gráfica de entre la resistencia a compresión y la relación agua-cemento para los cementos colombianos. (Sánchez De Guzmán, 2001). 
En la Figura 4 se presenta el resumen de los datos obtenidos para el ensayo a la flexión con carga en el centro de la luz, donde se puede notar el incremento de la resistencia con el incremento del contenido de fibras de acero.

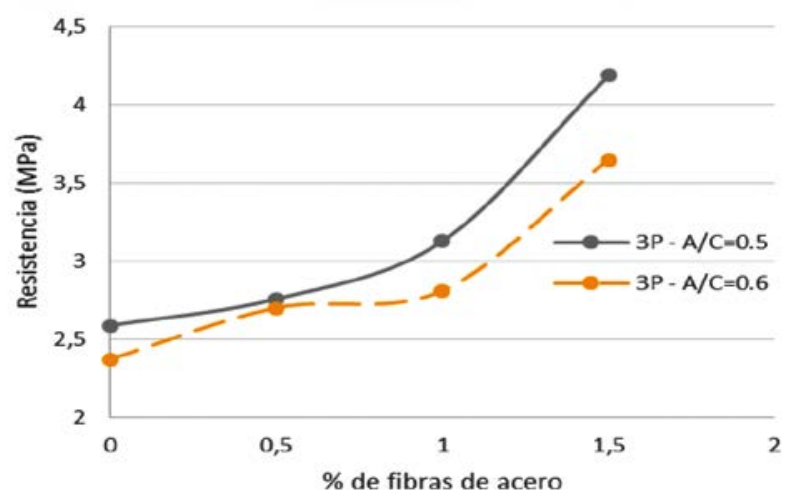

Figura 4. Variación de la resistencia a flexión con carga en el centro de la luz en función del contenido de fibras de acero. (Carrillo \& Díaz, 2014)

\section{Ensayo a Flexión con cargas a los tercios de la luz}

Para evaluar la resistencia a la flexión con cargas a los tercios de la luz se siguió el procedimiento establecido en la norma de ensayo INV E-414-07 (Invías, 2007), dicho montaje se puede observar en la Figura 5.

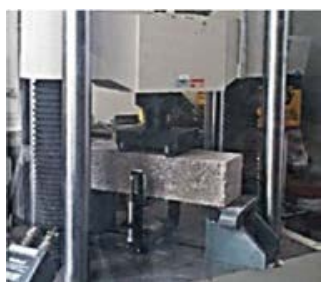

a)

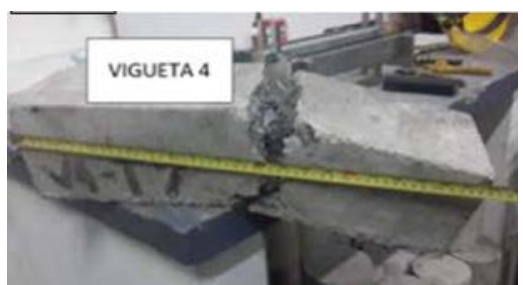

b)
Figura 5. Ensayo a flexión con cargas a los tercios de la luz. a) Montaje de las viguetas, b) Falla típica del concreto adicionado con fibras de acero.

El comportamiento de las curvas esfuerzo-deformación es muy similar al presentado para el caso de flexión en el centro de la luz.

En la Figura 6 se presenta el resumen de los datos obtenidos para el ensayo a la flexión con cargas a los tercios de la luz, donde se puede notar el incremento de la resistencia con el incremento del contenido de fibras de acero.

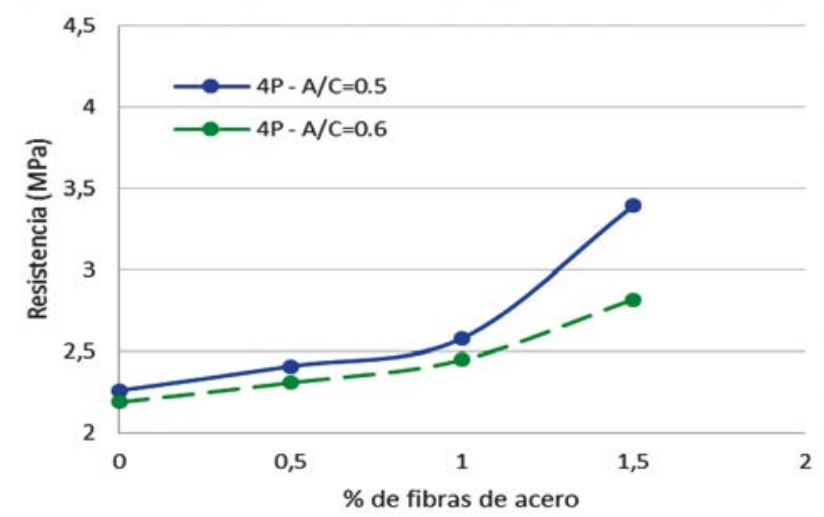

Figura 6. Variación de la resistencia a flexión con carga en los tercios de la luz en función del contenido de fibras de acero. (Carrillo \& Díaz, 2014)

\section{Ensayo a Tracción indirecta}

Para evaluar la resistencia a la tracción indirecta se siguió el procedimiento establecido en la norma de ensayo INV E-411-07 (Invías, 2007) (Figura 7).

a)

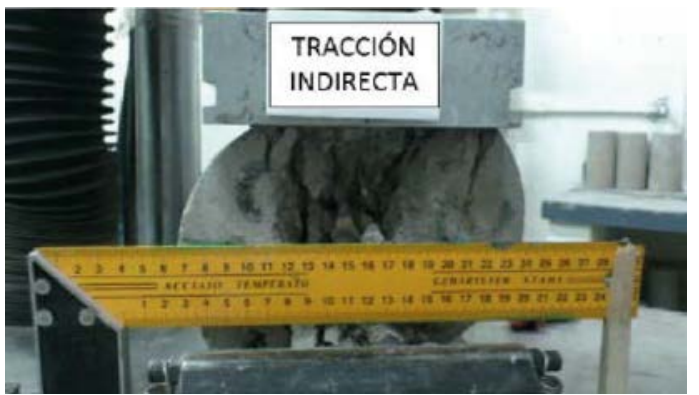

b)

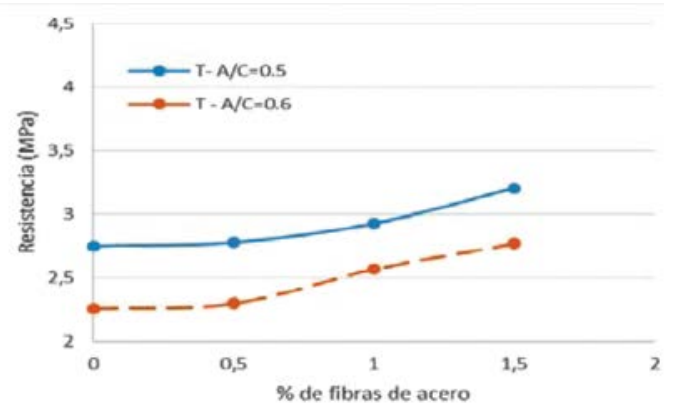

Figura 7. Ensayo a tracción indirecta. a) Forma de la falla b) Variación de la resistencia a la tracción, en función del contenido de fibras y la relación A/C. (Carrillo \& Díaz, 2014) 


\section{CONCLUSIONES}

Se realizó una caracterización de los tipos de fallas para asociarlas al ensayo, de lo cual se obtuvo el siguiente resultado. El ensayo a flexión con carga en el centro de la luz (3 puntos) tiene una predominancia por generar fisuras verticales, en el caso del ensayo a flexión con cargas en los tercios de la luz, los resultados son bastantes dispersos, sin permitir concluir nada.

El ensayo a flexión a tres puntos, muestra resistencias a la fractura inicial del $17 \%$ mayores, que el ensayo a 4 puntos en el concreto reciclado.

La resistencia a la compresión de los concretos con adición de agregado grueso reciclado, se ve afectada con base a los niveles teóricos que presentan los concretos en Colombia.

El uso de las dos metodologías para determinar la resistencia a la flexión de vigas de concreto con fibras, no afectan los módulos de rotura finales, ya que se obtienen valores similares para los dos ensayos.
Es claro que los concretos con la adición de fibras, proporcionan mayor ductilidad del material, convirtiéndose esto en una adecuada alternativa para solucionar los problemas de patologías que presentan los pavimentos de concreto sometidos a grandes cargas, como las que generan los medios de transporte masivo en la ciudad de Bogotá.

Usos potenciales de esta mezcla de concreto con reemplazo del agregado por reciclado de concreto se encuentran estructuras que sean sometidas a esfuerzos de flexión, como los son capas de rodadura de pavimentos, placas de contrapiso, placas aéreas y muros estructurales portantes de concreto.

\section{REFERENCIAS BIBLIOGRÁFICAS}

1. Carrillo, J., \& Díaz, L. (2014). Efecto de la adición de un tipo de fibras cortas de acero en la resistencia a la flexión del concreto con agregado reciclado. Tesis de pregrado, Universidad De La Salle. Bogotá.

2. Committe ACI 211. (2002). ACI 211.1. Standar practice for selecting proportions for normal, heavyweight, and mass concrete. Obtenido de https://www. academia.edu/6871828/Standard_Practice_for_Selecting_Proportions_for_Normal_Heavyweight_and_Mass_Concrete_ACI_211.1-91_Reported_by_ ACI_Committee_211_Committee_Members_Voting_on_1991_Revision_CONTENTS

3. Committe ACI 555. (2001). Removal and reuse of hardened concrete. ACI 555 R-01. Obtenido de http://bpesol.com/bachphuong/media/images/ book/555r_01.pdf

4. Icontec. (2010). Normas técnicas Colombianas NTC. Ensayo de resistencia a la compresión de especímenes de concreto NTC 673. Obtenido de https:// tienda.icontec.org/wp-content/uploads/pdfs/NTC673.pdf

5. INV E-414-07. (2007). Especificaciones generales de construcción de carreteras y normas de ensayo para materiales de carreteras. Resistencia a la flexión del concreto método de la viga simple cargada en los tercios de la luz

6. INV E-404-07. (2007). Especificaciones generales de construcción de carreteras y normas de ensayo para materiales de carreteras. Elaboración y curado en el laboratorio de muestras de concreto para ensayos de compresión y flexión.

7. INV E-415-07 (2007). Especificaciones generales de construcción de carreteras y normas de ensayo para materiales de carreteras. Resistencia a la flexión del concreto método de la viga simple cargada en el punto central.

8. INV E-414-07 (2007). Especificaciones generales de construcción de carreteras y normas de ensayo para materiales de carreteras. Ensayo de tracción indirecta de cilindros normales de concreto.

9. López-Gayarre, F., Serna, P., Domingo-Cabo, A., Serrano-López, M., \& López-Colina, C. (1994). Influenced of recycled aggregate quality and proportioning criteria on recycled concrete properties. Waste Management, 3022-3028.

10. Neha, V. S. (2013). Effect of Demolition Waste on Compressive Strength of Cement Matrix. Journal of Academia and Industrial Research (JAIR) 2.5, 295.

11. Rammamurthy, K., \& Gumaste, K. (1998). Properties of recycled aggregate concrete. Ind.Concr. J 72, 49-53.

12. Sánchez De Guzmán, D. (2001). Tecnología del concreto. Bogotá, Colombia: Bhandar Editores. 